Pacific Journal of Mathematics

ON POWER-INVARIANCE 


\title{
ON POWER-INVARIANCE
}

\author{
ELOISE HAMANN
}

Let $R$ be a commutative ring with identity, and consider the power series ring $R[[X]]$ in one analytic indeterminate over $R$. Is the coefficient ring $R$ unique in the sence that if $R[[X]]$ is isomorphic to $S[[Y]]$ with $Y$ an analytic indeterminate over $S$, need $S$ be isomorphic to $R$ ? Whenever this is the case, $R$ will be called power-invariant. It will be shown that if $R$ is a quasi-local or a complete semi-local ring then $R$ is powerinvariant.

The answer to the general question was not known by the author until the Commutative Algebra Conference in June of 1974 at the University of Nebraska where a counterexample was produced by Andy Magid. He has graciously requested that it be reproduced in this paper. The fact that rings with nilpotent Jacobson radical are power-invariant is known [6]. The paper will also show that if $R[[X]]=S[[Y]]$, under the assumption that certain elements are not zero-divisors that there exist one-to-one maps from $R$ into $S$ and $S$ into $R$. In particular, this is the case if $R$ is a domain. Finally, the paper generalizes the power-invariant results to an arbitrary number of variables.

The following notational conventions will be observed and referred to throughout the paper. $J(R)$ will denote the Jacobson radical of a ring $R$. $W, R, S, X, Y, j, u, v$ will be such that $W=R[[X]]=S[[Y]]$ where $X$ and $Y$ are analytic indeterminates over $R$ and $S$ respectively; $Y=j+u X$, and $X=k+v Y$ where $J \in J(R), u \in W, k \in J(S)$, and $v \in W$.

Note that since $W$ is complete with respect to both $(X)$ and $(Y)$, $W$ is also complete with respect to $(X, Y)$ and thus also complete with respect to the ideals $j W$ and $k W$ contained in $(X, Y)$. (If $\left\{w_{n}\right\}$ is a Cauchy sequence in the $(X, Y)$-adic topology, it can be written as a sum of two Cauchy sequences $\left\{s_{n}\right\}$ and $\left\{t_{n}\right\}$ which are also Cauchy with respect to the $(X)$-adic and $(Y)$-adic topologies respectively. Let $s$ be the limit of $\left\{s_{n}\right\}$ in the $(X)$-adic topology and $t$ the limit of $\left\{t_{n}\right\}$ in the $(Y)$-adic topology. Since $s$ and $t$ are then limit points of $\left\{s_{n}\right\}$ and $\left\{t_{n}\right\}$ respectively in the $(X, Y)$-adic topology, $s+t$ is a limit point of $\left\{w_{n}\right\}$. Conceivably the $(X, Y)$-adic topology may not be Hausdorff, so that limits aren't necessarily unique.) Further, $R$ is certainly a closed subset of $W$ in the $j W$-adic topology so $R$ is complete with respect to $(j)$, the ideal of $R$ generated by $j$, but perhaps not Hausdorff. Similarly, $S$ is complete but perhaps not 
Hausdorff with respect to $(k)$, the ideal of $S$ generated by $(k)$.

1. Andy Magid's counterexample. The example is the completion with respect to a certain ideal of Melvin Hochster's counterexample to the question whether $R[X]$ isomorphic to $S[Y]$ implies $S$ is isomorphic to $R$, where $X$ and $Y$ are ordinary indeterminates over $R$ and $S$ respectively. Specifically, there exists a Noetherian ring $R$ with zero Jacobson radical which has a finitely generated nonfree module $P$ such that $P \oplus R \cong R^{3}$. Taking symmetric algebras of both sides yields $A[T]$ isomorphic to $R[X, Y, Z]$ where $T,\{X, Y, Z\}$ are indeterminates over $A$ and $R$ respectively and $A$ is the symmetric algebra of $P$, and is not isomorphic to $R[X, Y]$. See either [3] or [4] for more details. If $M$ is a $B$-module, let $\widehat{S}_{B}(M)$ denote the complete symmetric algebra of $M$ over $B$, i.e., the completion of the symmetric algebra $S_{B}(M)$ with respect to the ideal generated by $M$. To get the counterexample for the power series case, take the complete symmetric algebras of $P \oplus R$ and $R^{3}$ over $R$. Then $\widehat{S}_{R}(P \oplus R) \cong$ $\hat{S}_{R}(P)[[T]]$ and $\hat{S}_{R}\left(R^{3}\right) \cong R[[X, Y, Z]]$ with $T$ an analytic indeterminate over $\hat{S}_{R}(P)$ and $X, Y, Z$ independent analytic indeterminates over $R$. It remains to show that $\widehat{S}_{R}(P)$ is not isomorphic to $R[[X, Y]]$. It suffices to show that $\widehat{S}_{R}(P) \cong R[[X, Y]]$ implies $S_{R}(P) \cong R[X, Y]$. Let $M$ be any finitely generated $R$-module. $J\left(\hat{S}_{R}(M)\right)$ is the ideal generated by $M$ since $M \hat{S}(M)$ is certainly contained in $J\left(\hat{S}_{R}(M)\right)$ and $\hat{S}(M) / M \hat{S}(M) \cong R$ whose Jacobson radical is zero. Thus, the associated graded ring of $\hat{S}(M)$ with respect to $\mathrm{J}(\hat{S}(M))$ is $\hat{S}(M) / M \hat{S}(M) \oplus$ $M \hat{S}(M) /] M \hat{S}(M)]^{2} \oplus \cdots$ which is isomorphic to $\hat{S}(M)$. Thus, $\hat{S}(M)$ determines $S(M)$ and the result follows.

2. Some power-invariant rings. The following theorem from [8] and [9] will be needed for the results of this section.

THEOREM 1. Let $B=\sum_{i=0}^{\infty} a_{i} X^{i} \in R[[X]]$, and suppose that $\phi$ is an $R$-endomorphism of $R[[X]]$ such that $\phi(X)=B$. Then:

(a) $\phi$ is onto if and only if $a_{1}$ is a unit of $R$.

(b) If $\phi$ is onto, then $\phi$ is one to one.

(c) $\phi$ is an automorphism if and only if $a_{1}$ is a unit of $R$.

THEOREM 2. Let $B=\sum_{i=0}^{\infty} b_{i} X^{i} \in R[[X]]$. If $\bigcap_{n=1}^{\infty}\left(b_{0}^{n}\right)=0$ in $R$ (or $\bigcap_{n=1}^{\infty}\left(B^{n}\right)=0$ in $R[[X]]$ ) and $R$ is complete with respect to $\left(b_{0}\right)$ (or $R[[X]]$ is complete with respect to $(B)$ ) then $\phi_{B}$ which maps $\sum_{i=0}^{\infty} a_{i} X^{i}$ to $\sum_{i=0}^{\infty} a_{i} B^{i}$ is an $R$-endomorphism.

TheOREM 3. Let $B=\sum_{i=0}^{\infty} b_{i} X^{i} \in R[[X]]$. Let $A$ be an ideal of R. If $b_{0} A=A$, then $A \subseteq \bigcap_{k=1}^{\infty}\left(B^{k}\right)$. 
Also recall that an element of $R[[X]]$ is invertible if its constant term is invertible [7].

We are ready for our first result.

THEOREM 4. If $R$ is quasi-local, $R$ is power-invariant.

Proof. Let the notation be as in the next-to-last paragraph of the introduction. If $u$ is an invertible element of $W$, then Theorem 1 implies $R[[Y]]=R[[X]]=S[[Y]]$ and $Y$ is an analytic indeterminate over $R$. Thus, $S \cong W /(Y) \cong R$. By symmetry we can reduce to the case where both $u$ and $v$ are in the maximal ideal of $W$. For $w \in W$, let $w_{i} \in S$ be such that $w=w_{0}+w_{1} Y+\cdots$. Taking the $Y$-coefficient of both sides of $Y=j+u X$ we get $1=j_{1}+u_{0} X_{1}+$ $u_{1} X_{0}=j_{1}+u_{0} X_{1}+u_{1} k . \quad u_{0}$ is in the maximal ideal since $u$ is, so $j_{1}$ is invertible. Similarly $k^{1}$, the $X$-coefficient of $k$ is invertible. Suppose for the moment that there is an $R$-endomorphism of $R[[X]]$ which takes $X$ to $k$, and an $S$-endomorphism which takes $Y$ to $j$. In this case Theorem 1 would imply $R[[k]]=R[[X]]=S[[Y]]$ with $k$ analytically independent over $R$, and $R \cong S /(k)[[Y]]$ with $Y$ an analytic indeterminate over $S /(k)$. Similarly, $S \cong R /(j)[[X]]$ with $X$ analytically independent over $R /(j)$. However, $(X, j)=(X, Y)=(k, Y)$ so that $S /(k) \cong W /(X, Y) \cong R /(j)$. This yield $R \cong S$. It remains only to show the desired endomorphisms exist. $W$ is certainly complete with respect to $(j)$ (or $(k)$ ), so by Theorem 2 and symmetry, the result will follow from the following proposition.

Proposition. Let $R[[X]]=S[[Y]]=W$ where $Y=j+u X$ and $X=k+v Y$ as above. If $u$ is in $J(W)$, then $\bigcap_{n=1}^{\infty}\left(j^{n}\right)=0$ as an ideal in $R$.

Proof. Since $\bigcap_{n=1}^{\infty}\left(Y^{n}\right)=0$, by Theorem 3 it suffices to show that $j\left[\bigcap_{n=1}^{\infty}\left(j^{n}\right)\right]=\bigcap_{n=1}^{\infty}\left(j^{n}\right)$. Let $A=\bigcap_{n=1}^{\infty}\left(j^{n}\right)$. First assume that $\operatorname{Ann}_{R} j \subseteq A$. Let $f \in A$ so that $f=j t$, for some $t_{1}$, and $f=j^{n} t_{n}$ for some $t_{n}$ given $n$. $j\left(t_{1}-j^{n-1} t_{n}\right)=0$ implies $t_{1}-j^{n-1} t_{n} \in A$, which further implies $t_{1} \in\left(j^{n-1}\right)$. Since $n$ was arbitrary $t_{1} \in A$. Thus, $f \in j A$ and $j A=A$. Now let us show that ${A n}_{R} j \leqq A$. It suffices to show that $f j^{i}=0$ implies $f \in(j)$. Let $f j^{i}=0$, in which case $f Y^{i}=$ $\sum_{h=1}^{i}\left(\begin{array}{l}i \\ h\end{array}\right) f j^{i-h} u^{h} X^{h}$. If we take the $Y^{i}$ coefficient of both sides, we get $f_{0}$ expressed as a sum of terms of the form $f_{a} j_{b_{1}} j_{b_{i-h}} u_{c_{1}} u_{c_{h}} X_{d_{1}} X_{d_{h}}$ where $a, b_{\alpha}, c_{\alpha}, d_{\alpha}$, are all integers, and $1 \leqq h \leqq i$. (Recall $w_{i}$ denotes the $Y^{i}$ coefficient in $S$ of $w$ in $S[[Y]]$.) Further, $a+\sum_{\alpha=1}^{i-h} b_{\alpha}+\sum_{\alpha=1}^{h} c_{a}+$ $\sum_{\alpha=1}^{h} d_{\alpha}=i$. If such a term involves $f_{0}$, i.e., $a=0$, then not all of the $b_{\alpha}, c_{\alpha}$, and $d_{\alpha}$ can be $\geqq 1$ since this would give $\sum_{\alpha=1}^{i-h} b_{\alpha}+\sum_{\alpha=1}^{h} c_{\alpha}+$ 
$\sum_{\alpha=1}^{h} d_{\alpha} \geqq(i-h)+h+h>i$. Thus, any term involving $f_{0}$ involves either $j_{0}, u_{0}$, or $X_{0}$ each of which is in $J(W)$. If a term does not involve $f_{0}$, then it must involve $X_{0}$ or $j_{0}$. (If not then $a+\sum_{\alpha=1}^{i=h} b_{\alpha}+$ $\sum_{\alpha=1}^{h} c_{\alpha}+\sum_{a=1}^{h} d_{\alpha} \geqq 1+(i-h)+h>i$.) Since $X_{0}=k$ and $j_{0} \in(k)$, we have $f_{0}=a f_{0}+b k$ where $a \in J(W)$. Since $1-a$ is invertible, $f_{0} \in(k) \subseteq(k, Y)=(j, X)$. However, $f \in R$ so $f \in(j)$ as required.

CorollaRY. Let $R[[X]]=S[[Y]], y=j+u X$, and $X=k+v Y$. Let $P$ be a maximal ideal of $R$, and $Q$ the maximal $i d e a l$ of $S$ such that $(P, X)=(Q, Y)$, then $\hat{R}_{P} \cong \hat{S}_{Q}$ where the completion can be taken with respect to the maximal ideals in question or with respect to (j) $R_{P}$ and $(k) S_{Q}$.

Proof. Let $M=(P, X)=(Q, Y)$, then $W_{M}$ can be thought of as a subring of $R_{P}[[X]]$ or $S_{Q}[[Y]]$. In either case the completion of $W_{M}$ with respect to $M$ is all of $\hat{R}_{P}[[X]]$ or $\hat{S}_{Q}[[Y]]$ where $\hat{R}_{P}$ is completion with respect to $P R_{P}$ and $\hat{S}_{Q}$ is completion with respect to $Q S_{Q}$. Thus, $\hat{R}_{P}[[X]] \cong \hat{S}_{Q}[[Y]]$ and the result follows from Theorem 4. The proof of the other completion is similar, this time complete $W_{M}$ with respect to $(X, Y)=(j, X)=(k, Y)$.

Theorem 5. If $R$ is a complete semi-local ring, $R$ is powerinvariant.

Proof. Let $R[[X]]=S[[Y]]=W$, then $W$ and $S$ are also complete semilocal. Since a complete semilocal ring is a direct sum of complete local rings, the result follows from the corollary.

3. Existence of one to one maps. The following lemmas are needed for our next result. With notation as in the introduction, we keep the convention that if $w \in W=R[[X]]=S[[Y]]$ that $w_{i}$ is the $Y^{i}$ coefficient in $S$ of $W$, and we let $w^{i}$ denote the $X^{i}$ coefficient in $R$ of $w$. Since $i=0$ and $i=1$ are the only cases of interest, there should be no confusion with exponents.

Define $\phi: R \rightarrow R$ by $\phi(r)=\left(r_{0}\right)^{0}$.

Lemma 1. With $\varnothing, R, S, W, X, Y$ as above and $Y=j+u X$, $X=k+v Y$, we have $(Y) \cap R \cong \operatorname{Ker} \phi \subseteq(j) . \quad((j)$ denotes the ideal of $R$ generated by $j$ ).

Proof. Since $Y_{0}=0$ the first containment is trivial. Now suppose $r \in R_{0}$ and $\left(r_{0}\right)^{0}=0$, then $r_{0}=t X$ and $r=t X+f Y$. Since $r=r^{0}$ $r=f^{0} y^{0}=f^{0} j$. 
LEMma 2. With everything as in Lemma 1, assume $\phi(j)$ is either zero or not a zero-divisor. Then $\operatorname{Ker} \phi=0$, or $\operatorname{Ker} \phi=(j)$.

Proof. If $\phi(j)=0, \operatorname{Ker} \phi=(j)$ by Lemma 1 . If $\phi(j) \neq 0$, let $f \in \operatorname{Ker} \phi . \quad f=a j$ also by Lemma $1 . \quad 0=\phi(f) \phi(a) \phi(j)$, which implies $\phi(a)=0$. Thus, $a=b j$ and it is clear that $f \in \bigcap_{n=1}^{\infty}\left(j^{n}\right)$. Since $\phi(j)=\left(u_{0}\right)^{0} v^{0} j$ is not a zero-divisor, $j$ is not a zero-divisor. Thus $j\left[\bigcap_{n=1}^{\infty}\left(j^{n}\right)\right]=\bigcap_{n=1}^{\infty}\left(j^{n}\right)=0$ by Theorem 3. Thus, $f=0$ and Ker $\phi=0$.

LEMMA 3. With notation as above, $j$ not a zero-divisor implies $k$ is not a zero-divisor.

Proof. $j$ not a zero-divisor implies $\{j, X\}$ is a $W$-sequence. Suppose $k$ is a zero-divisor, then some element of $S$ kills $k$, say $s k=0$. We get $s X=s v Y$. Now $s v \notin(x)$ since then $s$ would be a multiple of $Y$. However, $s X=s v j+s v u X$ makes $s v j$ a multiple of $X$ which is a contradiction. Thus, $k$ is not a zero-divisor.

TheOREM 6. Let $R[[X]]=S[[Y]], Y=j+u X, X=k+v Y$. If $j$ and $\phi(j)=\left(j_{0}\right)^{0}$ are not zero-divisors (unless 0$)$, then there exist 1 to 1 maps from $R$ into $S$ and from $S$ into $R$.

Proof. We need only consider the two cases of Lemma 2.

Case 1. $\operatorname{Ker} \phi=(j) \neq 0$.

In this case $R$ is actually isomorphic to $S$. Let $A=\operatorname{Im} \phi$. If $r \in R, r=r_{0}+w Y$ for some $w \in W$ and $r=r^{0}=\left(r_{0}\right)^{0}+w^{0} Y^{0}=\phi(r)+$ $w^{0} j$. Thus, $R=A+(j), \phi=\phi^{2}$, and $A \cap(j)=0$ all follow. $\mathrm{R}=A+$ ( $j)=A+j R$ implies $R=A+j A+\cdots+j^{n} A+j^{n+1} R$ for any $n$. $R$ is complete with respect to $(j)$ and also Hausdorff as in the proof of Lemma 2. Thus, $R=A[[j]]$. $j$ not a zero-divisor and $A \cap(j)=0$ together imply that $j$ is an analytic indeterminate over $A$ so $R \cong$ $A[[X]]$. We have $W=A[[k, Y]]$. We next show that $(Y) \cap A[[k]]=0$. Let $t \in(Y) \cap A[[k]] . \quad t=l y=\sum_{i=0}^{\infty} a_{i} k^{i}$. (Here $\alpha_{i} \in A$ and $i$ is an ordinary subscript.) $a_{0} \in(j) \cap A=0$ so $l y=\sum_{i=1}^{\infty} a_{i} k^{i}$. Taking constant terms in $S$ we get $0=\sum_{i=1}^{\infty}\left(a_{i}\right)_{0} k^{i}$. Since $k$ is not a zero-divisor $\sum_{i=1}^{\infty}\left(a_{i}\right)_{0} k^{i-1}=0$ and $\left(a_{1}\right)_{0} \in(k)$. Thus, $a_{1} \in(k, Y) \cap A=(j) \cap A=0$. By induction $a_{i}=0$ for all $i$ and $(Y) \cap A[[k]]=0$. But then $A[[k]] \cong$ $W /(Y) \cong S$. $k$ not a zero-divisor and $(k) \cap A \subseteq(j) \cap A=0$ imply $k$ is also an analytic indeterminate over $A$ and $S \cong A[[X]] \cong R$.

Case 2. $\operatorname{Ker} \phi=0$.

Clearly the map from $R$ to $S$ which takes $r$ to $r_{0}$ is $1-1$. Let 
$\phi$ denote the map from $S$ to $S$ which takes $s$ to $\left(s^{0}\right)_{0}$. Since $k$ is not a zero-divisor, $\phi(k)$ is zero or not a zero-divisor. Thus, by Lemma 2 $\operatorname{Ker} \phi=0$ or $\operatorname{Ker} \phi=(k)$. If $\operatorname{Ker} \phi=(k)$ the above argument with the role of $R$ and $S$ reversed yields $R \cong S$. If $\operatorname{Ker} \phi=0$, the map from $S$ to $R$ which takes $s$ to $s^{0}$ is $1-1$.

Note. There is the following analogue of Theorem 6. The proof is due to Nagata and appears in [1].

THEOREM 7. If $R$ is an integral domain and $R[X]=S[Y]$, then there exist injective homomorphisms of $R$ into $S$ and $S$ into $R$.

4. $n-$ Variable case. I believe the work on power-invariance to data has involved only one variable. It is natural to consider the following question. When can one conclude $S \cong R$, if there exists some $n$ such that $R\left[\left[X_{1}, \cdots, X_{n}\right]\right]=S\left[\left[Y_{1}, \cdots, Y_{n}\right]\right]$ where the $X_{i}$ and $Y_{i}$ are independent analytic indeterminates over $R$ and $S$ respectively. To wit, we give the following definition:

DEFINITION. $R$ will be called "forever power-invariant" provided $S \cong R$ whenever there is a positive integer $n$ such that $S\left[\left[X_{1}, \cdots\right.\right.$, $\left.\left.X_{n}\right]\right] \cong R\left[\left[X_{1}, \cdots, X_{n}\right]\right]$ where the $X_{i}$ are independent analytic indeterminates over $R$ and $S$.

Induction readily yields the following:

THeOREM 8. If $R$ is a quasi-local or a complete semi-local ring, then $R$ is forever power-invariant.

The next theorem generalizes the result that a ring with nilpotent Jacobson radical is power-invariant under the 1-variable definition. It also relaxes the nilpotent condition to a nil Jacobson radical.

THEOREM 9. If every element of $J(R)$ is nilpotent, then $R$ is forever power-invariant.

Proof. Let $R\left[\left[X_{1}, \cdots, X_{n}\right]\right]=S\left[\left[Y_{1}, \cdots, Y_{n}\right]\right]=W$. Let $X, Y$ denote the vectors $\left(X_{1}, \cdots, X_{n}\right)$ and $\left(Y_{1}, \cdots, Y_{n}\right) ; J, K$ vectors in $R^{n}$ and $S^{n}$ respectively such that $Y=J+X U, X=K+Y V$ where $U$ and $V$ are $n x n$ matrices with entries in $W$. Since $J(R)$ is nil, each component of the vector $J$ is nilpotent. The key point is that if $j$ is a nilpotent element of $R$, every coefficient in its expression as an element of $S[[Y]]$ is also nilpotent. Now, careful examination of $Y=J+$ $K U+Y V U$ yields $(1, \cdots, 1)=J_{1}+K U_{1}+\operatorname{diag} V_{0} U_{0}$ by taking the $Y_{i}$ coefficient of the $i$ th component of each side. Here $J_{1}$ denotes 
the result of this application to $J ; U_{1}$ is the $n x n$ matrix whose $i$ th column consists of the $Y_{i}$ coefficients of the $i$ th column of $U$; and $U_{0}\left(V_{0}\right)$ is the $n x n$ matrix of constant terms of $U(V)$. Since $J_{1}$ and $K$ have entries in $J(S)$, (the elements of $J_{1}$ being nilpotent) the elements of the diagonal of $V_{0} U_{0}$ are invertible. The same is, of course, then true for $V U$. By a similar scrutiny of the same, i.e., $Y=J+K U+$ $Y V U$, this time taking the $Y_{j}$ coefficient of the $i$ th component of both sides with $i \neq j$, we get the entries of $V U$ which are off the diagonal to be in $J(W)$. Thus, $V U$ has an invertible determinant, whence both $V$ and $U$ do, and both are invertible matrices. Thus, if the maps from $R[X]$ to $R[[X]]$ which take $R$ to $R$ and $X$ to $J+X U$ or $(X-J) U^{-1}$ can be extended to $\left.R[X]\right]$ in the natural way, they are clearly inverse maps, and thus $R$-automorphisms. In order for the extensions to be made $R[[X]]$ needs to be complete and Hausdorff with respect to the ideal generated by the images of the $\left\{X_{i}\right\}$. This is clear in the case that $X$ maps to $J+X U=Y . \quad R[[X]]$ is certainly complete with respect to $\left(j_{1}, \cdots, j_{n}, X_{1}, \cdots, X_{n}\right)$. Since the $j_{i}$ are nilpotent and $\bigcap_{k=1}^{\infty}\left(X_{1}, \cdots, X_{n}\right)^{k}=0, \bigcap_{k=1}^{\infty}\left(j_{1}, \cdots, j_{n}, X_{1}, \cdots, X_{n}\right)^{k}=0$ which is equivalent to $R[[X]]$ being Hausdorff with respect to $\left(j_{1}, \cdots, j_{n}, X_{1}, \cdots, X_{n}\right)$. Thus, the map taking $X$ to $(X-J) U^{-1}$ can also be extended. Thus, $R[[X]]=R[[Y]]$ and $R \cong U /(Y) \cong S$.

\section{REFERENCES}

1. S. Abhyankar, P. Eakin and W. Heinzer, On the uniqueness of the ring of coefficients in a polynomial ring, J. Algebra (to appear).

2. Bourbaki, Commutative Algebra, Addison Wesley. Translated from Hermann, Paris, 1972.

3. P. Eakin and W. Heinzer, A Cancellation Problem for Commutative Rings, Proc. Kansas Commutative Algebra Conference Springer-Verlag Lecture Notes in Math. No. 311, Berlin, 1973.

4. M. Hochster, Non-uniqueness of coefficient rings in a polynomial rings, Proc. Amer. Math. Soc., 34 (1972), 81-82.

5. I. Kaplansky, Commutative Rings, Boston, Allyn and Bacon, 1970.

6. J. H. Kim, Power-invariant rings, Pacific J. Math. (to appear).

7. M. Nagata, Local Rrings, New York, Interscience, 1962.

8. M. O'Malley, and C. Wood, R-Endomorphisms of $R[[X]]$, J. Algebra, 15 (1970), $314-327$.

9. M. O'Malley, $R$-Automorphisms of $R[[X]]$, Proc. London Math. Soc., 20 (1970), 60-78. 10. O. Zariski and P. Samuel, Commutative Algebra, Vol. II, Princeton, N.J., Van Nostrand, 1960.

Received December 27, 1974. The authors wishes to give special thanks to Andy Magid for the counterexample in the paper. She also wishes to thank Melvin Hochster for helpful conversations.

North Central College 



\section{PACIFIC JOURNAL OF MATHEMATICS}

\section{EDITORS}

RICHARD ARENS (Managing Editor) University of California

Los Angeles, California 90024

\section{R. A. BEAUMONT}

University of Washington Seattle, Washington 98105
J. DugundjI Department of Mathematics University of Southern Californı Los Angeles, California 90007

D. Gilbarg AND J. Milgram Stanford University Stanford, California 94305

\section{ASSOCIATE EDITORS}

E. F. BECKENBACH

B. H. NeUMANN

F. WOLF

K. YosHidA

\section{SUPPORTING INSTITUTIONS}

UNIVERSITY OF BRITISH COLUMBIA CALIFORNIA INSTITUTE OF TECHNOLOGY UNIVERSITY OF CALIFORNIA MONTANA STATE UNIVERSITY UNIVERSITY OF NEVADA NEW MEXICO STATE UNIVERSITY OREGON STATE UNIVERSITY UNIVERSITY OF OREGON OSAKA UNIVERSITY

\author{
UNIVERSITY OF SOUTHERN CALIFORNIA \\ STANFORD UNIVERSITY \\ UNIVERSITY OF TOKYO \\ UNIVERSITY OF UTAH \\ WASHINGTON STATE UNIVERSITY \\ UNIVERSITY OF WASHINGTON \\ AMERICAN MATHEMATICAL SOCIETY \\ NAVAL WEAPONS CENTER
}




\section{Pacific Journal of Mathematics}

\section{Vol. 61, No. $1 \quad$ November, 1975}

Jiří Adámek, V. Koubek and Věra Trnková, Sums of Boolean spaces represent every

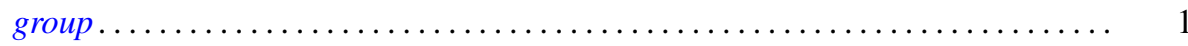

Richard Neal Ball, Full convex l-subgroups and the existence of $a^{*}$-closures of

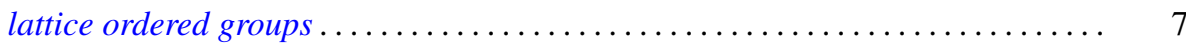

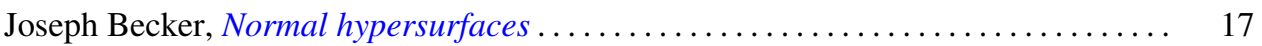

Gerald A. Beer, Starshaped sets and the Hausdorff metric . . . . . . . . . . . . . 21

Dennis Dale Berkey and Alan Cecil Lazer, Linear differential systems with

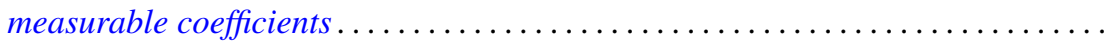

Harald Boehme, Glättungen von Abbildungen 3-dimensionaler

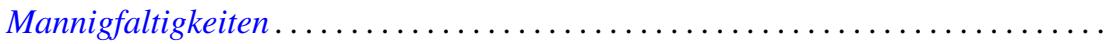

Stephen LaVern Campbell, Linear operators for which $T^{*} T$ and $T+T^{*}$

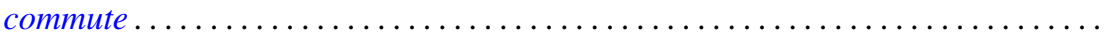

H. P. Dikshit and Arun Kumar, Absolute summability of Fourier series with

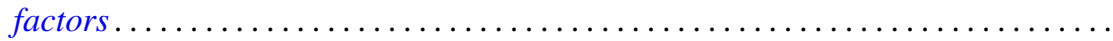

Andrew George Earnest and John Sollion Hsia, Spinor norms of local integral

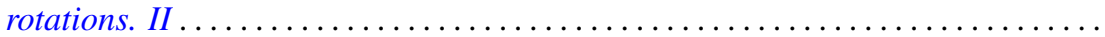

Erik Maurice Ellentuck, Semigroups, Horn sentences and isolic structures .........

Ingrid Fotino, Generalized convolution ring of arithmetic functions . . . . . . . . . . .

Michael Randy Gabel, Lower bounds on the stable range of polynomial rings .......

Fergus John Gaines, Kato-Taussky-Wielandt commutator relations and

characteristic curves

Theodore William Gamelin, The polynomial hulls of certain subsets of $C^{2}$

R. J. Gazik and Darrell Conley Kent, Coarse uniform convergence spaces. . .

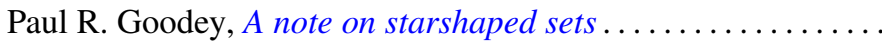

Eloise A. Hamann, On power-invariance

M. Jayachandran and M. Rajagopalan, Scattered compactification for $N \cup\{P\}$. . .

V. Karunakaran, Certain classes of regular univalent functions .

John Cronan Kieffer, A ratio limit theorem for a strongly subadditive set function in a locally compact amenable group .................

Siu Kwong Lo and Harald G. Niederreiter, Banach-Buck measure, density, and uniform distribution in rings of algebraic integers ........

Harold W. Martin, Contractibility of topological spaces onto metric spaces ....

Harold W. Martin, Local connectedness in developable spaces .

A. Meir and John W. Moon, Relations between packing and covering numbers of a tree.

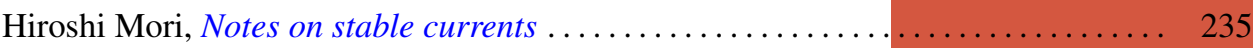

Donald J. Newman and I. J. Schoenberg, Splines and the logarithmic function . . . . 241

M. Ann Piech, Locality of the number of particles operator....

Fred Richman, The constructive theory of $K T$-modules .......

Gerard Sierksma, Carathéodory and Helly-numbers of

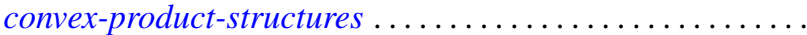

Raymond Earl Smithson, Subcontinuity for multifunctions .... . . 